\title{
Depth Enhancement of Optical Scanning Holography with a Spiral Phase Plate
}

\author{
Ni Chen, Zhenbo Ren, Antony Chan, Xing Sun, and Edmund Y. Lam* \\ Department of Electrical and Electronic Engineering, The University of Hong Kong, Pokfulam, Hong Kong. \\ *elam@eee.hku.hk
}

\begin{abstract}
A spiral phase plate is applied to the optical scanning holography system to improve the depth resolution of the reconstruction, the simulation results show that the depth interval can be resolved at a $0.4 \mu \mathrm{m}$ with only a single hologram.
\end{abstract}

OCIS codes: $100.3190,110.1758$.

\section{Theory}

Optical scanning holography (OSH) is a technique that records the holographic information of a three-dimensional scene by two-dimensional scanning [1]. The setup of the OSH is as Fig. 1 shows while the pupil $p_{2}$ is a pinhole. With this setup the optical transfer function (OTF) [1] is

$$
\begin{aligned}
H\left(k_{x}, k_{y} ; z\right)= & \exp \left[j \frac{z}{2 k}\left(k_{x}{ }^{2}+k_{y}{ }^{2}\right)\right] \\
& \times \iint_{-\infty}^{\infty} p_{1}{ }^{*}\left(x^{\prime}, y^{\prime}\right) p_{2}\left(x^{\prime}+x, y^{\prime}+y\right) \exp \left[j \frac{z}{f}\left(x^{\prime} k_{x}+y^{\prime} k_{y}\right)\right] d x^{\prime} d y^{\prime},
\end{aligned}
$$

where $p_{n}$ is the pupil function, $k_{x}, k_{y}$ are the spatial frequency coordinates, $f$ is the focal length of the lens, and $\lambda$ is the illumination wavelength.

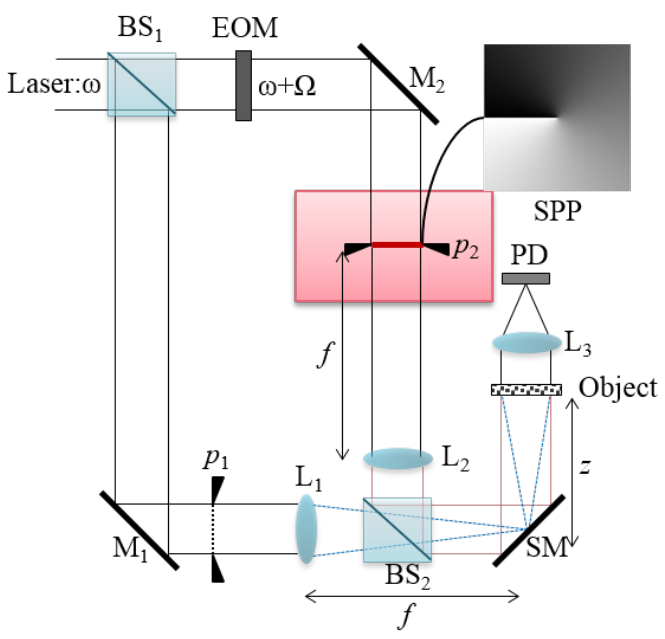

Fig. 1. Setup of the modified OSH system with a spiral phase plate (SPP).

The resolution of the OSH system is an important issue, especially the depth resolution. To improve it, there are many different techniques have been developed. On one hand, the resolution was enhanced by recording more information of the objects, such as the double detection method with different wavelengths (DW-OSH) [2], positions (DDOSH) [3] or pupils (DP-OSH) [4] changed in the detection process. On the other hand, the point spread function (PSF) of the OSH system is manipulated by designing the pupils $[5,6]$, since the resolution of an optical system is revealed by the characteristics of the Fresnel zone plate (FZP). Recently, SPP was used to achieve edge extraction in the OSH [7]. In this paper we propose a new way of using the SPP in the OSH and demonstrate that can enhance the depth resolution of the OSH, which we call it SPP-OSH. In the proposed SPP-OSH system, the pinhole in the conventional setup 
is replaced by an SPP. The SPP can be a digital holographic element (DOE) or spatial light modulator (SLM) which acts as an SPP. With this setup, zone plate pattern in the scanning mirror plane (SM in Fig. 1) is the interferometric intensity of a spherical beam and a vortex beam. The spiral phase function is $l \theta$, where $l$ is the topological charge and $(r, \theta)$ is polar coordinate. We consider $l=1$ in this paper. The vortex wave after $p_{2}$ becomes

$$
h_{s p p}(r, \theta)=\operatorname{circ}\left(\frac{r}{R}\right) \exp (i \theta)
$$

and the Fourier transform of Eq. (2) is

$$
H_{s p p}(\rho, \varphi)=\frac{\pi R}{j 2 \rho}\left[H_{0}\left(\frac{2 \pi R \rho}{\lambda f}\right) J_{0}\left(\frac{2 \pi R \rho}{\lambda f}\right)-H_{1}\left(\frac{2 \pi R \rho}{\lambda f}\right) J_{1}\left(\frac{2 \pi R \rho}{\lambda f}\right)\right] \exp (j \varphi),
$$

where $(\rho, \varphi)$ is polar coordinate, $\lambda$ is the wavelength and $f$ is the focal length of the lens. $J_{n}$ is the n-th order Bessel function, $H_{n}$ is the $n$-th order Struve function. By substituting Eq. (2) and Eq. (3) into Eq. (1), we have

$$
H\left(k_{x}, k_{y} ; z\right)=\exp \left[-j \frac{z}{2 k} \rho^{2}\right] \times H_{s p p}\left(-\frac{z}{f} \rho,-\arctan (\varphi)\right) .
$$

From Eq. (4), it can be observed that the transfer function in the SPP-OSH system is the combination of the conventional transfer function and the transfer function of the SPP.

\section{Simulation Results}

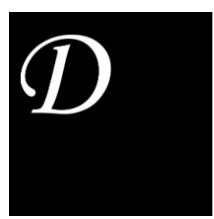

(a)

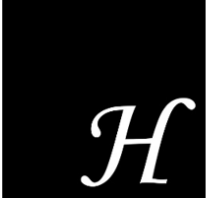

(b)

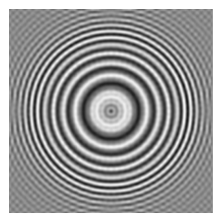

(c)

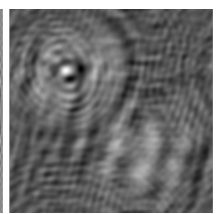

(d)

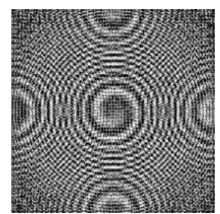

(e)

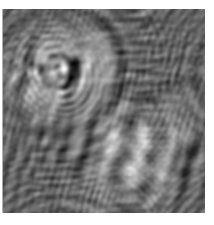

(f)

Fig. 2. Object with two plane images located at (a) $z_{1}$ and (b) $z_{2}$ : Sample of fringe patterns with the (c) conventional OSH and the proposed (e) SPP-OSH; Holograms recorded with the (d) conventional OSH and the proposed (f) SPP-OSH.

In the simulation, we compared the proposed method to the conventional OSH and the DW-OSH. Fig. 2(a) and (b) are the two plane images of the object used in the simulation. The two images were separated along the optical axis with a distance of $\Delta z$. The wavelength of the illumination laser is $\lambda=532 \mathrm{~nm}$, the other wavelength in the DW-OSH is $\lambda=632 \mathrm{~nm}$. The scanning step is $0.1 \mu \mathrm{m}$. Fig. 2(c) and (e) are the zone patterns of the conventional OSH or DWOSH and SPP-OSH respectively, and Fig. 2(d) and (f) are the holograms of the conventional OSH or DW-OSH and SPP-OSH respectively. In the hologram generation, the system contains white Gaussian noise at $30 \mathrm{~dB}$.

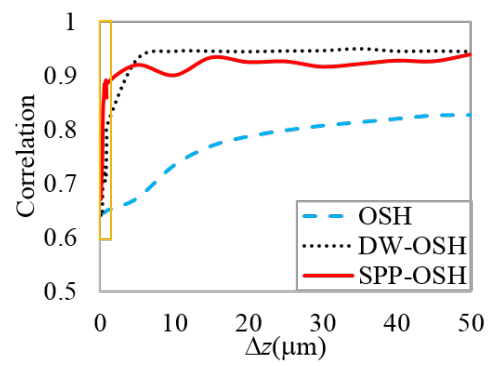

(a)

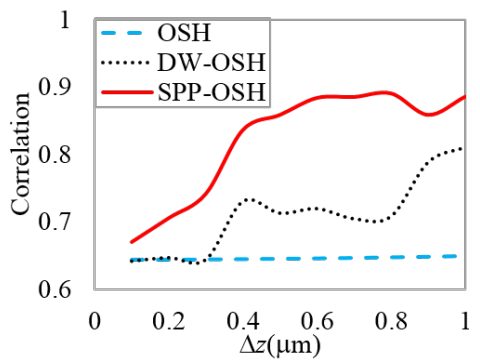

(b)

Fig. 3. The correlation between the reconstructed images and the original images in terms of the depth interval of the object planes.

Block Jacobi-type restrictive preconditioned conjugate gradient(BJ-PRPCG) [2,8] algorithm was used to reconstruct the sectional images from the holograms. Fig. 3(a) shows the correlation coefficients of the reconstructed images versus 
the depth distance between the object image planes. The blue dashed line is the conventional OSH, the black dotted line is the DW-OSH, and the red solid line is the proposed SPP-OSH. Fig. 3(b) is the magnified image of the yellow square area in Fig. 3(a). It can be observed that the depth resolution of the DW-OSH and the SPP-OSH is much better than the conventional OSH, and the SPP-OSH is better than the DW-OSH. However, the DW-OSH get better correlation coefficient when the depth interval of the object planes is larger. If we define the depth resolution as the distance interval in the object planes when the correlation of the reconstructed images is larger than 0.8 , the depth resolutions of the OSH, DW-OSH and the SPP-OSH are $30 \mu \mathrm{m}, 1 \mu \mathrm{m}$ and $0.4 \mu \mathrm{m}$ respectively. The threshold is chosen as 0.8 since the conventional OSH starts changing very slowly while the value is larger than 0.8 in our simulation. Fig. 4(a-c) are the reconstructed images with the conventional OSH, DW-OSH and SPP-OSH while the depth distance of the object image planes is $30 \mu \mathrm{m}$ and Fig. 4(d-f) are the reconstructions while the depth distance of the object planes is $0.4 \mu \mathrm{m}$. The depth resolution of the proposed method is much better than the conventional OSH and the DW-OSH.

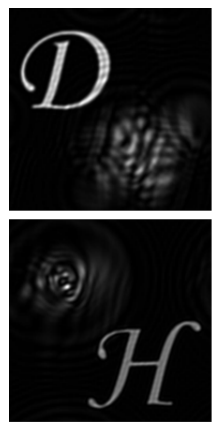

(a)

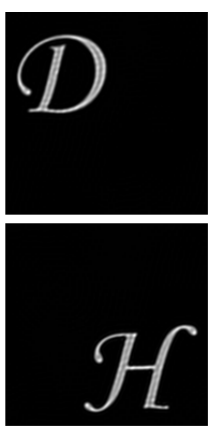

(b)

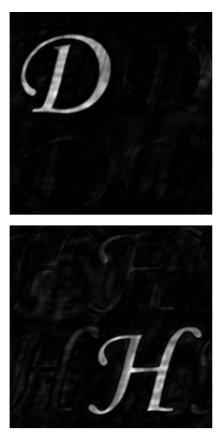

(c)

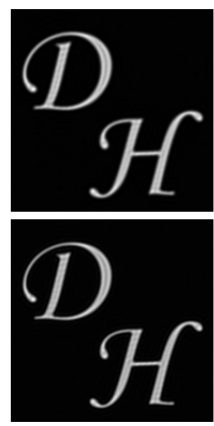

(d)

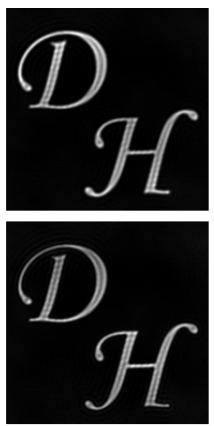

(e)

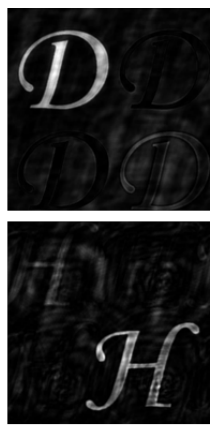

(f)

Fig. 4. Reconstructed sectional images while (a-c) $\Delta z=30 \mu \mathrm{m}$ and (d-f) $\Delta z=0.4 \mu \mathrm{m}$. (a)(d) are the OSH method, (b)(e) are the DW-OSH method, and (c)(f) are the SPP-OSH method.

\section{Summary}

The SPP increases the number of the zone plates, and reduces the width of the outermost zone, therefore enhances the depth resolution in the OSH. In our simulation, it reaches even $0.4 \mu \mathrm{m}$ with only a single hologram.

\section{Acknowledgment}

This work was supported in part by the Research Grants Council of the Hong Kong Special Administrative Region, China, under Projects HKU 7138 \11E and 7131 \12E, and by the NSFC $\backslash$ RGC under Project N_HKU714 \13.

\section{References}

1. T.-C. Poon, Optical Scanning Holography with MATLAB (Springer, 2007).

2. J. Ke, T.-C. Poon, and E. Y. Lam, "Depth resolution enhancement in optical scanning holography with a dualwavelength laser source," Applied Optics 50, H285-H296 (2011).

3. H. Ou, T.-C. Poon, K. K. Y. Wong, and E. Y. Lam, "Depth resolution enhancement in double-detection optical scanning holography," Applied Optics 52, 3079-3087 (2013).

4. H. Ou, T.-C. Poon, K. K. Y. Wong, and E. Y. Lam, "Enhanced depth resolution in optical scanning holography using a configurable pupil,” Photonics Research 2, 64-70 (2014).

5. G. Indebetouw, W. Zhong, and D. Chamberlin-Long, "Point-spread function synthesis in scanning holographic microscopy," Journal of the Optical Society of America A 23, 1708-1717 (2006).

6. T.-C. Poon and K. B. Doh, "On the theory of optical hilbert transform for incoherent objects," Optics Express 15, 3006-3011 (2007).

7. Y. Pan, W. Jia, J. Yu, K. Dobson, C. Zhou, Y. Wang, and T.-C. Poon, "Edge extraction using a time-varying vortex beam in incoherent digital holography," Optics Letters 39, 4176-4179 (2014).

8. X. Zhang, E. Y. Lam, and T.-C. Poon, "Reconstruction of sectional images in holography using inverse imaging," Optics Express 16, 17,215-17,226 (2008). 